\title{
Lebesgue points and the fundamental convergence theorem for superharmonic functions on metric spaces
}

\author{
Anders Björn, Jana Björn and Mikko Parviainen
}

\begin{abstract}
We prove the nonlinear fundamental convergence theorem for superharmonic functions on metric measure spaces. Our proof seems to be new even in the Euclidean setting. The proof uses direct methods in the calculus of variations and, in particular, avoids advanced tools from potential theory. We also provide a new proof for the fact that a Newtonian function has Lebesgue points outside a set of capacity zero, and give a sharp result on when superharmonic functions have $L^{q}$-Lebesgue points everywhere.
\end{abstract}

\section{Introduction}

Our main objective is to provide a new proof for the fundamental convergence theorem of nonlinear potential theory in $\mathbb{R}^{n}$ and on metric measure spaces. By the fundamental convergence theorem, a regularized infimum of superharmonic functions is superharmonic provided that it is locally uniformly bounded from below. Furthermore, the regularization changes the limit only on a set of capacity zero.

During the last decade, analysis and potential theory on metric measure spaces have been developing rapidly. Heinonen-Koskela [24] introduced upper gradients as a substitute for the modulus of the usual gradient and Koskela-MacManus [38] extended the concept to weak upper gradients. In Shanmugalingam [42], Sobolev type spaces (called Newtonian spaces) on

2000 Mathematics Subject Classification: Primary: 31C45; Secondary: 31C05, 35J60. Keywords: $\mathcal{A}$-harmonic, fundamental convergence theorem, Lebesgue point, metric space, Newtonian function, nonlinear, $p$-harmonic, quasicontinuous, Sobolev function, superharmonic, superminimizer, supersolution, weak upper gradient. 
metric measure spaces were defined as the collection of $p$-integrable functions with $p$-integrable upper gradients. See also Cheeger [13] for an alternative approach which leads to the same spaces.

Newtonian spaces enable us to study variational integrals and potential theoretic models can be built on minimizers of the $p$-Dirichlet integral

$$
\int g_{u}^{p} d \mu
$$

where $g_{u}$ denotes the minimal $p$-weak upper gradient of $u$. This generalizes the Euclidean potential theory based on equations of $p$-Laplace type as in Heinonen-Kilpeläinen-Martio [23], as well as potential theory on Riemannian manifolds, Heisenberg groups, Carnot-Carathéodory spaces and graphs, see e.g. Hajłasz-Koskela [18]. For recent developments in potential theory on metric spaces, see e.g. Shanmugalingam [43, 44], BjörnMacManus-Shanmugalingam [12], J. Björn [10, 11], Kinnunen-Shanmugalingam [36, 37], Kinnunen-Martio [33, 34, 35], Björn-Björn-Shanmugalingam [6, 7], A. Björn [1, 2, 3] and Björn-Björn [4]. For a nice general overview of the theory we refer to Heinonen [20].

In $\mathbb{R}^{n}$, the nonlinear fundamental convergence theorem dates back to the 1988 paper of Heinonen and Kilpeläinen [21]. See also Heinonen-Kilpeläinen [22] as well as Kilpeläinen [27], and for the classical result Doob [15]. Their proofs rely on advanced tools from potential theory, whereas our proof is based on direct methods in the calculus of variations. We establish directly in Theorem 7.1 that a decreasing sequence of superminimizers is a superminimizer provided, of course, that a suitable lower bound exists. The Lebesgue differentiation theorem for Newtonian functions then implies that by changing the superminimizer on a set of capacity zero we obtain a superharmonic function, and a truncation argument completes the proof.

Our approach has one important advantage compared to the existing versions in the Euclidean literature: superminimizers in $\mathbb{R}^{n}$ belong to the usual Sobolev spaces with a.e.-equivalence classes whereas Newtonian functions are defined up to sets of capacity zero and have Lebesgue points outside of a set of capacity zero. This follows from the results in Shanmugalingam [42] and from Kinnunen-Latvala [30], where a discrete maximal function was used to prove the existence of Lebesgue points for representatives of Hajłasz-Sobolev functions. We seize the opportunity to provide a shorter and more direct proof for Newtonian spaces based on upper gradients, see Theorem 4.1. In Section 6 we give several results concerning Lebesgue points of superminimizers and superharmonic functions.

The fundamental convergence theorem is a basic tool in the theory of balayage: it implies several fundamental properties of the balayage in a 
straightforward manner, see Björn-Björn-Mäkäläinen-Parviainen [5]. The theory of balayage in turn plays an essential role in the study of regular boundary points, capacity and polar sets, see [5] for some of these applications on metric spaces.

Acknowledgement. We would like to thank Takayori Ono for pointing out Theorem 7.3 to us.

\section{Preliminaries}

We assume throughout the paper that $1<p<\infty$ and that $X=(X, d, \mu)$ is a metric space endowed with a metric $d$ and a positive complete Borel measure $\mu$ such that $0<\mu(B)<\infty$ for all open balls $B \subset X$. Assume also that $\Omega \subset X$ is nonempty and open. (To avoid pathologies we also assume that $X$ contains at least two points.)

The measure $\mu$ is doubling if there exists a constant $C_{\mu} \geq 1$, such that for all balls $B=B\left(x_{0}, r\right):=\left\{x \in X: d\left(x, x_{0}\right)<r\right\}$ in $X$,

$$
\mu(2 B) \leq C_{\mu} \mu(B)
$$

where $\lambda B=B\left(x_{0}, \lambda r\right)$.

In this paper, a path in $X$ is a rectifiable nonconstant continuous mapping from a compact interval to $X$. A path can thus be parametrized by arc length $d s$. We also make the convention that $|\infty-\infty|=|-\infty-(-\infty)|=\infty$.

We follow Heinonen-Koskela [24] introducing upper gradients as follows (they called them very weak gradients).

Definition 2.1. A nonnegative Borel function $g$ on $X$ is an upper gradient of an extended real-valued function $f$ on $X$ if for all paths $\gamma:\left[0, l_{\gamma}\right] \rightarrow X$,

$$
\left|f(\gamma(0))-f\left(\gamma\left(l_{\gamma}\right)\right)\right| \leq \int_{\gamma} g d s
$$

If $g$ is a nonnegative measurable function on $X$ and if (2.1) holds for $p$-a.e. path, then $g$ is a $p$-weak upper gradient of $f$.

By saying that (2.1) holds for $p$-a.e. path, we mean that it fails only for a path family with zero $p$-modulus, see Definition 2.1 in Shanmugalingam [42]. It is implicitly assumed that $\int_{\gamma} g d s$ is defined (with a value in $[0, \infty]$ ) for p-a.e. path.

The $p$-weak upper gradients were introduced in Koskela-MacManus [38]. They also showed that if $g \in L^{p}(X)$ is a $p$-weak upper gradient of $f$, then one can find a sequence $\left\{g_{j}\right\}_{j=1}^{\infty}$ of upper gradients of $f$ such that $g_{j} \rightarrow g$ in 
$L^{p}(X)$. If $f$ has an upper gradient in $L^{p}(X)$, then it has a minimal $p$-weak upper gradient $g_{f} \in L^{p}(X)$ in the sense that for every $p$-weak upper gradient $g \in L^{p}(X)$ of $f, g_{f} \leq g$ a.e., see Corollary 3.7 in Shanmugalingam [43].

Next we define a version of Sobolev spaces on the metric space $X$ due to Shanmugalingam in [42]. Cheeger [13] gave an alternative definition which leads to the same space, when $p>1$.

Definition 2.2. Whenever $u \in L^{p}(X)$, let

$$
\|u\|_{N^{1, p}(X)}=\left(\int_{X}|u|^{p} d \mu+\inf _{g} \int_{X} g^{p} d \mu\right)^{1 / p},
$$

where the infimum is taken over all upper gradients of $u$. The Newtonian space on $X$ is the quotient space

$$
N^{1, p}(X)=\left\{u:\|u\|_{N^{1, p}(X)}<\infty\right\} / \sim,
$$

where $u \sim v$ if and only if $\|u-v\|_{N^{1, p}(X)}=0$.

Definition 2.3. The capacity of a set $E \subset X$ is the number

$$
C_{p}(E)=\inf \|u\|_{N^{1, p}(X)}^{p},
$$

where the infimum is taken over all $u \in N^{1, p}(X)$ such that $u=1$ on $E$.

The capacity is countably subadditive. For this and other properties as well as equivalent definitions of the capacity we refer to Kilpeläinen-Kinnunen-Martio [29] and Kinnunen-Martio [31, 32].

We say that a property holds quasieverywhere (q.e.) if the set of points for which the property does not hold has capacity zero. The capacity is the correct gauge for distinguishing between two Newtonian functions. Indeed, if $u \in N^{1, p}(X)$, then $u \sim v$ if and only if $u=v$ q.e. Moreover, if $u, v \in N^{1, p}(X)$ and $u=v$ a.e., then $u \sim v$.

We need a Newtonian space with zero boundary values defined as follows. For an open set $\Omega \subset X$,

$$
N_{0}^{1, p}(\Omega)=\left\{\left.f\right|_{\Omega}: f \in N^{1, p}(X) \text { and } f=0 \text { in } X \backslash \Omega\right\} .
$$

One can replace the assumption " $f=0$ in $X \backslash \Omega$ " with " $f=0$ q.e. in $X \backslash \Omega$ " without changing the space.

Definition 2.4. We say that $X$ supports a $(q, p)$-Poincaré inequality if there exist constants $C>0$ and $\lambda \geq 1$ such that for all balls $B \subset X$, all integrable functions $f$ on $X$ and for all upper gradients $g$ of $f$,

$$
\left(f_{B}\left|f-f_{B}\right|^{q} d \mu\right)^{1 / q} \leq C(\operatorname{diam} B)\left(f_{\lambda B} g^{p} d \mu\right)^{1 / p},
$$

where $f_{B}:=f_{B} f d \mu:=\int_{B} f d \mu / \mu(B)$. 
In the definition of the Poincaré inequality we can equivalently assume that $g$ is a $p$-weak upper gradient.

Hölder's inequality implies that if $X$ supports a $(q, p)$-Poincaré inequality, then it supports a $(q, t)$-Poincaré inequality for every $t>p$. If $\mu$ is doubling and $X$ supports a $(1, p)$-Poincaré inequality, then $X$ also supports a $(q, p)$ Poincaré inequality for some $q>p$, see Hajłasz-Koskela [18]. If moreover $X$ is complete and $p>1$, then $X$ supports a $(1, \bar{p})$-Poincaré inequality for some $\bar{p}<p$, by Keith-Zhong [26].

If $X$ supports a $(1, p)$-Poincaré inequality and $\mu$ is doubling, it follows that Lipschitz functions are dense in $N^{1, p}(X)$, see Shanmugalingam [42]. If $X$ is also complete, then the functions in $N^{1, p}(X)$ are quasicontinuous, see Björn-Björn-Shanmugalingam [8]. This means that in the Euclidean setting, $N^{1, p}\left(\mathbb{R}^{n}\right)$ is the refined Sobolev space, considered e.g. in Chapter 4 in Evans-Gariepy [16]. At the beginning of Section 5 we add some general assumptions valid throughout the rest of the paper.

Unless otherwise stated, the letter $C$ denotes various positive constants whose exact values are unimportant and may vary with each usage. Recall also that $f_{+}=\max \{f, 0\}$ and $f_{-}=\max \{-f, 0\}$.

We end this section by showing that as long as $u$ is real-valued a.e., it makes no difference how we interpret the inequality (2.1) in the special case when the left-hand side is either $|\infty-\infty|$ or $|(-\infty)-(-\infty)|$. Our main interest is in $N^{1, p}$ (and $N_{\mathrm{loc}}^{1, p}$ ) functions, and such functions are necessarily real-valued a.e.

Observe that Proposition 2.5 as well as the results in Section 3 hold in general metric spaces. In particular we do not assume that $\mu$ is doubling nor that any Poincaré inequality is satisfied. That $X$ is complete will only be assumed from Section 5 onwards.

Proposition 2.5. Let $u$ be a function which is finite a.e. and assume that $g$ is such that for $p$-a.e. path $\gamma:\left[0, l_{\gamma}\right] \rightarrow X$ it is true that either

$$
|u(\gamma(0))|=\left|u\left(\gamma\left(l_{\gamma}\right)\right)\right|=\infty \quad \text { or } \quad\left|u(\gamma(0))-u\left(\gamma\left(l_{\gamma}\right)\right)\right| \leq \int_{\gamma} g d s .
$$

Then $g$ is a p-weak upper gradient of $f$.

Proof. Let $\Gamma$ be the set of the exceptional paths $\gamma$ for which (2.3) does not hold for some subpath of $\gamma$, and let $\Gamma^{\prime}=\{\gamma: \gamma \subset E\}$, where $E=\{x \in X$ : $|u(x)|=\infty\}$. Since $(2.3)$ holds for $p$-a.e. path and $\mu(E)=0$, it follows that the path family $\Gamma \cup \Gamma^{\prime}$ is of zero $p$-modulus, see Shanmugalingam [42], Proposition 3.1 and Lemma 3.2. 
Let $\gamma$ be a path which is not in $\Gamma \cup \Gamma^{\prime}$. Then there is $t \in\left[0, l_{\gamma}\right]$ such that $\gamma(t) \notin E$. Therefore

$$
\begin{aligned}
\left|u(\gamma(0))-u\left(\gamma\left(l_{\gamma}\right)\right)\right| & \leq|u(\gamma(0))-u(\gamma(t))|+\left|u(\gamma(t))-u\left(\gamma\left(l_{\gamma}\right)\right)\right| \\
& \leq \int_{\gamma \mid[0, t]} g d s+\int_{\left.\gamma\right|_{\left[t, l_{\gamma}\right]}} g d s=\int_{\gamma} g d s
\end{aligned}
$$

since the second alternative in (2.3) holds for $\left.\gamma\right|_{[0, t]}$ and $\left.\gamma\right|_{\left[t, l_{\gamma}\right]}$. We have thus shown that $g$ is a $p$-weak upper gradient of $u$.

\section{Consequences of Fuglede's and Mazur's lemmas}

In this section, we show that if a sequence is bounded in $N^{1, p}(X)$ and converges q.e., then the limit is in $N^{1, p}(X)$, see Corollary 3.3 , a fact that will be essential in our proof of Lemma 7.2. To accomplish this, we first utilize the boundedness of the sequence to extract a weakly converging subsequence whose weak upper gradients converge weakly. Mazur's lemma then allows us to pass from the weak to the strong convergence in $L^{p}$. This is the content of the proof of Lemma 3.2. In Proposition 3.1, we use Fuglede's lemma and Proposition 2.5 to show that there exists a function that differs from the strong limit at most on a set of measure zero and belongs to $N^{1, p}(X)$. Furthermore, the strongly converging sequence converges to this limit q.e., and therefore it coincides with the original limit q.e. This proves that the original limit belongs to $N^{1, p}(X)$.

Proposition 3.1. Assume that $f_{j} \in N^{1, p}(X)$ and that $g_{j} \in L^{p}(X)$ is a $p$ weak upper gradient of $f_{j}, j=1,2, \ldots$. Assume further that $f_{j} \rightarrow f$ and $g_{j} \rightarrow g$ in $L^{p}(X)$, and that $g$ is nonnegative. Then there is a function $\tilde{f}=f$ a.e. such that $g$ is a p-weak upper gradient of $\tilde{f}$, and thus $\tilde{f} \in N^{1, p}(X)$. There is also a subsequence $\left\{f_{j_{k}}\right\}_{k=1}^{\infty}$ such that $f_{j_{k}} \rightarrow \tilde{f}$ q.e. in $X$.

Moreover, if either $f \in N^{1, p}(X)$ or there is a subsequence $\left\{f_{j_{k}}\right\}_{k=1}^{\infty}$ such that $f_{j_{k}} \rightarrow f$ q.e., then we may choose $\tilde{f}=f$.

Proof. By passing to a subsequence if necessary, we may assume that $f_{j} \rightarrow f$ a.e., and by Fuglede's lemma (see Shanmugalingam [42], Lemma 3.4 and Remark 3.5) that $\int_{\gamma} g_{j} d s \rightarrow \int_{\gamma} g d s \in \mathbb{R}$ for all $\gamma \notin \Gamma$, where $\Gamma$ is a path family with zero $p$-modulus. We concentrate on paths $\gamma$ such that neither $\gamma$ nor any of its subpaths belong to $\Gamma$, and such that $g_{j}$ is an upper gradient for $f_{j}$ along $\gamma$ for every $j=1,2, \ldots$. This holds for $p$-a.e. path, cf. the proof of Proposition 2.5.

Let $\tilde{f}=\limsup _{j \rightarrow \infty} f_{j}$, and observe that $\tilde{f}$ is defined at every point of $X$ and $\tilde{f}=f$ a.e. in $X$. Let $E=\{x \in X:|\tilde{f}(x)|=\infty\}$. We see that 
either $\gamma(0), \gamma\left(l_{\gamma}\right) \in E$ or

$$
\begin{aligned}
\left|\tilde{f}(\gamma(0))-\tilde{f}\left(\gamma\left(l_{\gamma}\right)\right)\right| & \leq \limsup _{j \rightarrow \infty}\left|f_{j}(\gamma(0))-f_{j}\left(\gamma\left(l_{\gamma}\right)\right)\right| \\
& \leq \limsup _{j \rightarrow \infty} \int_{\gamma} g_{j} d s=\int_{\gamma} g d s .
\end{aligned}
$$

As $\mu(E)=0$, Proposition 2.5 shows that $g$ is indeed a $p$-weak upper gradient of $\tilde{f}$.

Let now $\hat{f}=\liminf _{j \rightarrow \infty} f_{j}$. Arguing exactly as above we see that $g$ is also a $p$-weak upper gradient of $\hat{f} \in N^{1, p}(X)$ and that $\hat{f}=f=\tilde{f}$ a.e. in $X$. As $\hat{f}, \tilde{f} \in N^{1, p}(X)$, it follows that $\hat{f}=\tilde{f}$ q.e., and thus $f_{j} \rightarrow \tilde{f}$ q.e. in $X$.

On the other hand, if $f \in N^{1, p}(X)$, then $\hat{f}=f=\tilde{f}$ a.e. implies that $f=\tilde{f}$ q.e. and $f \sim \tilde{f}$ in $N^{1, p}(X)$ so that $g$ is a $p$-weak upper gradient also of $f$. Moreover, $f_{j} \rightarrow f$ q.e. in $X$.

Finally, if $f_{j_{k}} \rightarrow f$ q.e., then again $f=\tilde{f}$ q.e., and $g$ is a $p$-weak upper gradient also of $f$.

Lemma 3.2. Assume that $g_{j}$ is a $p$-weak upper gradient of $u_{j}, j=1,2, \ldots$, and that both sequences $\left\{u_{j}\right\}_{j=1}^{\infty}$ and $\left\{g_{j}\right\}_{j=1}^{\infty}$ are bounded in $L^{p}(X)$. Then there are $u, g \in L^{p}(X)$, convex combinations $v_{j}=\sum_{i=j}^{N_{j}} a_{j, i} u_{i}$ with p-weak upper gradients $\bar{g}_{j}=\sum_{i=j}^{N_{j}} a_{j, i} g_{i}$ and a subsequence $\left\{u_{j_{k}}\right\}_{k=1}^{\infty}$, such that

(a) both $u_{j_{k}} \rightarrow u$ and $g_{j_{k}} \rightarrow g$ weakly in $L^{p}(X)$;

(b) both $v_{j} \rightarrow u$ and $\bar{g}_{j} \rightarrow g$ in $L^{p}(X)$;

(c) $v_{j} \rightarrow u$ q.e.;

(d) $g$ is a p-weak upper gradient of $u$.

Proof. Since $L^{p}(X)$ is reflexive, there is a subsequence of $\left\{u_{j}\right\}_{j=1}^{\infty}$ which converges weakly in $L^{p}(X)$. Taking a subsequence of this subsequence, we obtain a sequence, again denoted $\left\{u_{j}\right\}_{j=1}^{\infty}$, such that both $\left\{u_{j}\right\}_{j=1}^{\infty}$ and $\left\{g_{j}\right\}_{j=1}^{\infty}$ converge weakly in $L^{p}(X)$ say to $v$ and $g$ (where $g$ is not necessarily a $p$-weak upper gradient of $v$ ). As $g_{j}, j=1,2, \ldots$, are nonnegative we may choose $g$ nonnegative.

Applying Mazur's lemma (see, e.g., Yosida [45, pp. 120-121]), we find convex combinations $v_{j}^{\prime}=\sum_{i=j}^{N_{j}^{\prime}} a_{i, j}^{\prime} u_{i}$ converging strongly to $v$ in $L^{p}(X)$ as $j \rightarrow \infty$. Furthermore, $g_{j}^{\prime}=\sum_{i=j}^{N_{j}^{\prime}} a_{i, j}^{\prime} g_{i}$ is a $p$-weak upper gradient of $v_{j}^{\prime}$. Since $g_{j}^{\prime} \rightarrow g$ weakly in $L^{p}(X)$, we can again apply Mazur's lemma to obtain convex combinations $v_{j}=\sum_{i=j}^{N_{j}} a_{i, j} u_{i}$ with $p$-weak upper gradients $\bar{g}_{j}=\sum_{i=j}^{N_{j}} a_{i, j} g_{i}$ such that $v_{j} \rightarrow v$ and $\bar{g}_{j} \rightarrow g$ in $L^{p}(X)$. By Proposition 3.1, there exists $u=v$ a.e. such that $g$ is a weak upper gradient of $u$ and $v_{j} \rightarrow u$ q.e. 
If the sequence in Lemma 3.1 converges q.e., then the limit belongs to $N^{1, p}(X)$. We also obtain an estimate for the minimal $p$-weak upper gradient of the limit.

Corollary 3.3. Assume that $\left\{u_{i}\right\}_{i=1}^{\infty}$ is bounded in $N^{1, p}(X)$ and that $u_{i} \rightarrow u$ q.e. Then $u \in N^{1, p}(X)$ and

$$
\int_{X} g_{u}^{p} d \mu \leq \liminf _{i \rightarrow \infty} \int_{X} g_{u_{i}}^{p} d \mu .
$$

Proof. There exists a subsequence $\left\{u_{i_{j}}\right\}_{j=1}^{\infty}$ such that

$$
\lim _{j \rightarrow \infty} \int_{X} g_{u_{i_{j}}}^{p} d \mu=\liminf _{i \rightarrow \infty} \int_{X} g_{u_{i}}^{p} d \mu
$$

By Lemma 3.2, there are convex combinations $v_{j}$ of $\left\{u_{i_{j}}\right\}_{j=1}^{\infty}$ and functions $\tilde{u}, g \in L^{p}(X)$ such that $v_{j} \rightarrow \tilde{u}$ q.e., $g$ is a $p$-weak upper gradient of $\tilde{u}$ and, after possibly taking another subsequence, both $u_{i_{j}} \rightarrow \tilde{u}$ and $g_{u_{i_{j}}} \rightarrow g$ weakly in $L^{p}(X)$.

Since $v_{j}$ tends to $u$ q.e., it follows that $\tilde{u}=u$ q.e., and thus $g$ is also a $p$-weak upper gradient of $u$. Since $g_{u_{i_{j}}} \rightarrow g$ weakly in $L^{p}(X)$, we have

$$
\int_{X} g_{u}^{p} d \mu \leq \int_{X} g^{p} d \mu \leq \lim _{j \rightarrow \infty} \int_{X} g_{u_{i_{j}}}^{p} d \mu=\liminf _{i \rightarrow \infty} \int_{X} g_{u_{i}}^{p} d \mu
$$

\section{Lebesgue points of $N^{1, p}$-functions}

In this section we prove that Newtonian functions have Lebesgue points q.e. in $X$. To accomplish this, we estimate the Newtonian norm of the function

$$
\tilde{u}(x)=\limsup _{j \rightarrow \infty}\left(f_{B\left(x, 2^{-j}\right)}|u|^{q} d \mu\right)^{1 / q}
$$

in terms of the Newtonian norm of $u$. The proof utilizes the noncentred maximal function in an essential way. In particular, the upper gradient of $\tilde{u}$ is expressed in terms of the maximal function of the upper gradient of $u$ itself. The definition of capacity and the $L^{p}$-boundedness of the maximal function then give an estimate for the capacity of the set where $\tilde{u}$ is large. To complete the proof, we add and subtract a Lipschitz function in the definition of Lebesgue points and use the fact that, under our assumptions, Lipschitz functions are dense in Newtonian spaces. 
We assume that $\mu$ is doubling with doubling constant $C_{\mu}$ and that $X$ supports a $(1, \bar{p})$-Poincaré inequality for some $\bar{p}<p$. By iterating the doubling condition, it follows with $s=\log _{2} C_{\mu}$ and $C=C_{\mu}^{-2}$ that

$$
\frac{\mu(B(z, r))}{\mu(B(y, R))} \geq C\left(\frac{r}{R}\right)^{s}
$$

for all balls $B(y, R) \subset X, z \in B(y, R)$ and $0<r \leq R<\infty$. However, the choice $s=\log _{2} C_{\mu}$ may not be optimal, and we just assume that $s$ is any number such that (4.1) is satisfied.

Let $q_{0}=s p /(s-p)$ if $s>p$ and $q_{0}=\infty$ if $s \leq p$. For every exponent $q<q_{0}$, we show that every Newtonian function has $L^{q}$-Lebesgue points outside a set of capacity zero. In particular, since $q_{0}>p$, this holds with exponent $p$.

Theorem 4.1. If $u \in N_{\text {loc }}^{1, p}(X)$ and $q<q_{0}$, then for q.e. $x \in X$,

$$
\lim _{r \rightarrow 0} f_{B(x, r)}|u-u(x)|^{q} d \mu=0 .
$$

In particular, q.e. $x \in X$ is a Lebesgue point of $u$.

For Hajłasz spaces on doubling metric spaces, Kinnunen and Latvala proved that every Hajłasz-Sobolev function has a representative which has Lebesgue points q.e., see Theorem 4.5 in [30]. Their result covers Theorem 4.1 in the case when $X$ is complete, as then Newtonian functions are quasicontinuous and coincide with the above best representatives of Hajłasz-Sobolev functions, see Theorem 4.9 in Shanmugalingam [42] and Theorem 1.1 in Björn-Björn-Shanmugalingam [8]. We seize the opportunity to provide a shorter and more direct proof of Theorem 4.1 in Newtonian spaces which also covers the case when $X$ is not complete. The proof utilizes the following two notions.

Definition 4.2. For $f \in L_{\mathrm{loc}}^{1}(X)$, the noncentred maximal function is

$$
M^{*} f(x):=\sup _{B} f_{B}|f| d \mu
$$

where the supremum is taken over all balls $B$ containing $x$.

Definition 4.3. For $u \in L_{\text {loc }}^{q}(X), q \geq 1$, let

$$
\begin{aligned}
u_{j}(x) & =\left(f_{B\left(x, 2^{-j}\right)}|u|^{q} d \mu\right)^{1 / q}, \quad j=0,1, \ldots, \\
T_{q} u(x) & =\limsup _{j \rightarrow \infty} u_{j}(x) .
\end{aligned}
$$


It is easy to see that the noncentred maximal function is comparable to the usual Hardy-Littlewood maximal function. The following maximal function theorem is well known, see e.g. Theorem 2.2 in Heinonen [19].

Theorem 4.4. Let $t>1$. If $f \in L^{t}(X)$, then $M^{*} f \in L^{t}(X)$ and

$$
\int_{X}\left(M^{*} f\right)^{t} d \mu \leq C \int_{X}|f|^{t} d \mu
$$

where $C$ depends only on the doubling constant $C_{\mu}$ and on $t$. For $f \in L^{1}(X)$, the following weak type estimate holds for all $\tau>0$,

$$
\mu\left(\left\{x \in X: M^{*} f(x)>\tau\right\}\right) \leq \frac{C}{\tau} \int_{X}|f| d \mu,
$$

where $C$ depends only on the doubling constant $C_{\mu}$.

Lemma 4.5. Let $g$ be an upper gradient of a function $u \in L^{q}(X)$ and assume that a $(q, \bar{p})$-Poincaré inequality holds for $u$ and $g$. Then $C\left(M^{*} g^{\bar{p}}\right)^{1 / \bar{p}}$ is a p-weak upper gradient of $T_{q} u$, where $C$ depends only on $C_{\mu}, q, \bar{p}$ and the constants in the Poincaré inequality.

Proof. Since $T_{q} u \leq\left(M^{*}|u|^{q}\right)^{1 / q}$, Theorem 4.4 implies that $T_{q} u<\infty$ a.e. Let $\gamma:\left[0, l_{\gamma}\right] \rightarrow X$ be a path (parametrized by arc length) such that the set $\left\{\tau \in\left[0, l_{\gamma}\right]: T_{q} u(\gamma(\tau))=\infty\right\}$ has zero (one-dimensional) Lebesgue measure. This holds for $p$-a.e. path, see the proof of Lemma 3.2 in Shanmugalingam [42].

We first show that $C\left(M^{*} g^{\bar{p}}\right)^{1 / \bar{p}}$ is an upper gradient of $u_{j}$ along $\gamma$ for all sufficiently large $j$. Let $j$ be such that $r_{j}=2^{-j} \leq 2 l_{\gamma}$. By splitting $\gamma$ into parts if necessary, we can assume that $r_{j} / 2 \leq l_{\gamma} \leq r_{j}$. Let $x=\gamma(0)$ and $y=\gamma\left(l_{\gamma}\right)$ be the endpoints of $\gamma$. Since $u \in L^{q}(X)$, both $u_{j}(x)$ and $u_{j}(y)$ are finite. The $(q, \bar{p})$-Poincaré inequality implies that for all $z \in B:=B\left(x, 2 r_{j}\right)$,

$$
\begin{aligned}
\left|u_{j}(x)-u_{j}(y)\right| & \leq\left|\left(f_{B\left(x, r_{j}\right)}|u|^{q} d \mu\right)^{1 / q}-u_{B}\right|+\left|\left(f_{B\left(y, r_{j}\right)}|u|^{q} d \mu\right)^{1 / q}-u_{B}\right| \\
& \leq\left(f_{B\left(x, r_{j}\right)}\left|u-u_{B}\right|^{q} d \mu\right)^{1 / q}+\left(f_{B\left(y, r_{j}\right)}\left|u-u_{B}\right|^{q} d \mu\right)^{1 / q} \\
& \leq C\left(f_{B}\left|u-u_{B}\right|^{q} d \mu\right)^{1 / q} \\
& \leq C r_{j}\left(f_{\lambda B} g^{\bar{p}} d \mu\right)^{1 / \bar{p}} \\
& \leq C r_{j}\left(M^{*} g^{\bar{p}}\right)^{1 / \bar{p}}(z) .
\end{aligned}
$$


As $\gamma \subset B$ and $l_{\gamma} \geq r_{j} / 2$, we get that

$$
\left|u_{j}(x)-u_{j}(y)\right| \leq C \int_{\gamma}\left(M^{*} g^{\bar{p}}\right)^{1 / \bar{p}} d s,
$$

i.e. $C\left(M^{*} g^{\bar{p}}\right)^{1 / \bar{p}}$ is an upper gradient of $u_{j}$ along $\gamma$.

Glueing together all the parts of $\gamma$ if necessary and assuming that either $T_{q} u(x)<\infty$ or $T_{q} u(y)<\infty$, we get

$$
\left|T_{q} u(x)-T_{q} u(y)\right| \leq \sup _{j}\left|u_{j}(x)-u_{j}(y)\right| \leq \int_{\gamma} C\left(M^{*} g^{\bar{p}}\right)^{1 / \bar{p}} d s,
$$

where the supremum is taken over sufficiently large $j$. Proposition 2.5 then shows that $C\left(M^{*} g^{\bar{p}}\right)^{1 / \bar{p}}$ is a $p$-weak upper gradient of $T_{q} u$.

Lemma 4.6. Let $q<q_{0}$. If $u \in N_{0}^{1, p}(B)$ for some ball $B \subset X$, then $T_{q} u \in N^{1, p}(X)$ and $\left\|T_{q} u\right\|_{N^{1, p}(X)} \leq C\|u\|_{N^{1, p}(B)}$, where $C$ depends only on $B, C_{\mu}, q, p$ and the constants in the Poincaré inequality.

Proof. If $s<p$, then (4.1) holds with $s$ replaced by $p$, so we can assume that $s \geq p$. Find $\bar{p}>p s /(p+s)$ so that $X$ supports a $(1, \bar{p})$-Poincaré inequality and so that $q s /(q+s)<\bar{p}<p \leq s$. Further, find $\varepsilon>0$ such that $\bar{q}:=q(1+\varepsilon)<s \bar{p} /(s-\bar{p})$ and $\bar{q}>p$. Since $\bar{q}<s \bar{p} /(s-\bar{p})$ and $\mu$ is doubling, $X$ supports a $(\bar{q}, \bar{p})$-Poincaré inequality as shown by Hajłasz and Koskela in Theorem 5.1 of [18].

Note that $\operatorname{supp} T_{q} u \subset \bar{B}$ and $T_{q} u \leq\left(M^{*}|u|^{q}\right)^{1 / q}$ on $\bar{B}$. By these facts, Theorem 4.4, and Sobolev's inequality, see Proposition 3.1 in J. Björn [10], we have that

$$
\begin{aligned}
\left(\int_{X}\left|T_{q} u\right|^{\bar{q}} d \mu\right)^{1 / \bar{q}} & \leq\left(\int_{X}\left(M^{*}|u|^{q}\right)^{1+\varepsilon} d \mu\right)^{1 / \bar{q}} \\
& \leq C\left(\int_{B}|u|^{\bar{q}} d \mu\right)^{1 / \bar{q}} \leq C\|u\|_{N^{1, p}(B)} .
\end{aligned}
$$

As $\operatorname{supp} T_{q} u \subset \bar{B}$ and $\bar{q}>p$, this implies that

$$
\left\|T_{q} u\right\|_{L^{p}(X)} \leq C\|u\|_{N^{1, p}(B)} .
$$

Let $g$ be an upper gradient of $u$. By Lemma $4.5, C\left(M^{*} g^{\bar{p}}\right)^{1 / \bar{p}}$ is a $p$-weak upper gradient of $T_{q} u$ and as $p / \bar{p}>1$, Theorem 4.4 together with (4.3) finishes the proof.

The following corollary follows directly from the definition of capacity and Lemma 4.6 since $T_{q} u(x) / \tau$ is admissible for calculating the capacity of $\left\{x \in X: T_{q} u(x)>\tau\right\}$. 
Corollary 4.7. If $u \in N_{0}^{1, p}(B)$ for some ball $B \subset X$, then for all $\tau>0$,

$$
C_{p}\left(\left\{x \in X: T_{q} u(x)>\tau\right\}\right) \leq \frac{C}{\tau^{p}}\|u\|_{N^{1, p}(X)}^{p} .
$$

Proof of Theorem 4.1. As Lebesgue points are a local issue, multiplying $u$ by a cut-off function, we can assume that $u \in N_{0}^{1, p}(B)$ for some ball $B \subset X$. There exist Lipschitz functions $u_{k}, k=1,2, \ldots$, such that $u_{k} \rightarrow u$ both in $N^{1, p}(X)$ and pointwise q.e. This was shown by Shanmugalingam in Corollary 3.9 and Theorem 4.1 of [42]. Writing

$$
|u-u(x)| \leq\left|u-u_{k}\right|+\left|u_{k}-u_{k}(x)\right|+\left|u_{k}(x)-u(x)\right|
$$

and using the fact that $u_{k}$ has Lebesgue points everywhere, we have for all $x \in X$ and all $k=1,2, \ldots$, that

$$
\limsup _{j \rightarrow \infty}\left(f_{B\left(x, 2^{-j}\right)}|u-u(x)|^{q} d \mu\right)^{1 / q} \leq T_{q}\left(u-u_{k}\right)(x)+\left|u_{k}(x)-u(x)\right| .
$$

The last term on the right-hand side tends to zero as $k \rightarrow \infty$ for q.e. $x \in X$. To estimate the first term on the right-hand side, we have by Corollary 4.7 for every $\tau>0$,

$$
C_{p}\left(\left\{x \in X: T_{q}\left(u-u_{k}\right)(x)>\tau\right\}\right) \leq \frac{C}{\tau^{p}}\left\|u-u_{k}\right\|_{N^{1, p}(X)}^{p} .
$$

This estimate and (4.4) imply that

$$
\begin{aligned}
C_{p}(\{x \in X & \left.\left.: \limsup _{j \rightarrow \infty}\left(f_{B\left(x, 2^{-j}\right)}|u-u(x)|^{q} d \mu\right)^{1 / q}>2 \tau\right\}\right) \\
& \leq C_{p}\left(\left\{x \in X:\left|u_{k}(x)-u(x)\right|>\tau\right\}\right)+\frac{C}{\tau^{p}}\left\|u-u_{k}\right\|_{N^{1, p}(X)}^{p} \rightarrow 0,
\end{aligned}
$$

as $k \rightarrow \infty$. The doubling property of $\mu$ then implies that for q.e $x \in X$,

$$
\lim _{r \rightarrow 0} f_{B(x, r)}|u-u(x)| d \mu \leq C \limsup _{j \rightarrow \infty} f_{B\left(x, 2^{-j}\right)}|u-u(x)| d \mu=0 .
$$

If $u \in N_{\text {loc }}^{1, p}(X)$ and $C_{p}(\{x\})>0$, then $x$ is a Lebesgue point for $u$ (and (4.2) holds), by Theorem 4.1. In the case when $p>s$ (and $X$ is complete) we can show that this is the case for all points and also improve upon Theorem 5.1 in Shanmugalingam [42], showing not only that every Newtonian function has a Hölder continuous representative, but that all representatives are Hölder continuous.

Proposition 4.8. Assume that $X$ is complete, $p>s$ and $x \in X$. Then $C_{p}(\{x\})>0$. 
Proof. Let $B \in X$ be a ball containing $x$ such that $X \backslash \overline{6 B} \neq \varnothing$. By Lemma 3.3 in J. Björn [10] and Theorem 1.1 in Kallunki-Shanmugalingam [25], it is sufficient to show that

$$
\inf _{u} \int_{B} g_{u}^{p} d \mu>0
$$

where the infimum is taken over $u \in \operatorname{Lip}(X)$ with $u(x)=1$ and $u=0$ in $X \backslash B$. By Theorem 5.1 in Hajłasz-Koskela [18], for such $u$, we have that

$$
\int_{B} g_{u}^{p} d \mu=\int_{10 \lambda B} g_{u}^{p} d \mu \geq C \sup _{y \in 2 B}\left|u(y)-u_{2 B}\right|,
$$

where $C$ is independent of $u$ and $\lambda \geq 1$ is the dilation constant in the Poincaré inequality. It follows from the Poincaré inequality that there is some $z \in 2 B \backslash B$. As $u(z)=0$ and $u(x)=1$, the supremum on the righthand side is always at least $\frac{1}{2}$. Hence the infimum in (4.5) is positive.

Theorem 4.9. Assume that $X$ is complete, $p>s$ and $u \in N^{1, p}(X)$. Then $u$ is $(1-s / p)$-Hölder continuous and all points are Lebesgue points for $u$.

Proof. By Theorem 5.1 in Shanmugalingam [42], there is $\tilde{u} \sim u$ which is $(1-s / p)$-Hölder continuous. As all points have positive capacity we must have $u=\tilde{u}$. Hence all points are Lebesgue points for $u$ (which also follows directly from Theorem 4.1 together with Proposition 4.8).

\section{Superminimizers and superharmonic functions}

In the rest of the paper, we assume that $X$ is complete, that $\mu$ is doubling, and that $X$ supports a $(1, p)$-Poincaré inequality. A deep theorem of KeithZhong [26] then shows that $X$ even supports a $(1, \bar{p})$-Poincaré inequality for some $\bar{p}<p$.

We say that $f \in N_{\text {loc }}^{1, p}(\Omega)$ if for every $x \in \Omega$ there exists $r_{x}$ such that $f \in N^{1, p}\left(B\left(x, r_{x}\right)\right)$. This is clearly equivalent to saying that $f \in N^{1, p}(V)$ for every open $V \Subset \Omega$. By saying that $V \Subset \Omega$ we mean that $\bar{V}$ is a compact subset of $\Omega$.

Definition 5.1. A function $u \in N_{\mathrm{loc}}^{1, p}(\Omega)$ is a minimizer in $\Omega$ if for all $\varphi \in$ $N_{0}^{1, p}(\Omega)$ we have

$$
\int_{\varphi \neq 0} g_{u}^{p} d \mu \leq \int_{\varphi \neq 0} g_{u+\varphi}^{p} d \mu .
$$

A function $u \in N_{\text {loc }}^{1, p}(\Omega)$ is a superminimizer in $\Omega$ if (5.1) holds for all nonnegative $\varphi \in N_{0}^{1, p}(\Omega)$, and a subminimizer in $\Omega$ if (5.1) holds for all nonpositive $\varphi \in N_{0}^{1, p}(\Omega)$. 
By Proposition 3.2 in A. Björn [2] it is enough to test (5.1) with (all, nonnegative and nonpositive, respectively) $\varphi \in \operatorname{Lip}(X)$ with $\operatorname{supp} \varphi \Subset \Omega$.

We shall use the ess lim inf-regularization

$$
u^{*}(x)=\operatorname{ess} \lim _{y \rightarrow x} \inf u(y)=\lim _{R \rightarrow 0} \underset{B(x, R)}{\operatorname{essinf}} u .
$$

For the reader's convenience let us verify that $u^{*}$ is indeed lower semicontinuous: Let $x \in \Omega$ and $a<u^{*}(x)$. Then there is $r>0$ such that $B(x, r) \subset \Omega$ and $\operatorname{essinf}_{B(x, r)} u>a$. It follows that $u^{*}(y)>a$ for all $y \in B(x, r)$. Letting $a \rightarrow u^{*}(x)$ we see that $u^{*}(x) \leq \liminf _{y \rightarrow x} u^{*}(y)$, and hence $u^{*}$ is lower semicontinuous.

Theorem 5.2. Let $u$ be a superminimizer in $\Omega$. Then

$$
u^{*}=u \quad \text { q.e. in } \Omega \text {. }
$$

This result was obtained by Kinnunen-Martio [33, Theorem 5.1], using a weak Harnack inequality for superminimizers. Here we give an alternative proof based on a supremum estimate for subminimizers, which is easier to obtain. The proof applies in the Euclidean setting as well. For a parabolic counterpart, see Kuusi [39].

Proof. By Theorem 4.1,

$$
E=\left\{x_{0} \in \Omega: \lim _{R \rightarrow 0} f_{B\left(x_{0}, R\right)}\left|u\left(x_{0}\right)-u\right| d \mu=0 \text { and }\left|u\left(x_{0}\right)\right|<\infty\right\}
$$

differs from $\Omega$ only in a set of capacity zero. Choose $x_{0} \in E$ and observe that $\left(u\left(x_{0}\right)-u\right)_{+}$is a nonnegative subminimizer. By the supremum estimate from Theorem 4.2 and Remark 4.4(2) in Kinnunen-Shanmugalingam [36], we have for $B\left(x_{0}, R\right) \Subset \Omega$ that

$$
\operatorname{essip}_{B\left(x_{0}, R / 2\right)}\left(u\left(x_{0}\right)-u\right)_{+} \leq C f_{B\left(x_{0}, R\right)}\left(u\left(x_{0}\right)-u\right)_{+} d \mu .
$$

Let $\varepsilon>0$. Since $x_{0} \in E$, there exists $R_{0}>0$ such that

$$
f_{B\left(x_{0}, R\right)}\left(u\left(x_{0}\right)-u\right)_{+} d \mu \leq f_{B\left(x_{0}, R\right)}\left|u\left(x_{0}\right)-u\right| d \mu<\varepsilon \quad \text { for all } 0<R<R_{0} .
$$

We deduce that

$$
C \varepsilon>\operatorname{ess}_{B\left(x_{0}, R / 2\right)}\left(u\left(x_{0}\right)-u\right)_{+} \geq \operatorname{ess~sup}_{B\left(x_{0}, R / 2\right)}\left(u\left(x_{0}\right)-u\right)=u\left(x_{0}\right)-\operatorname{essinf}_{B\left(x_{0}, R / 2\right)} u .
$$

Since this holds for every $0<R<R_{0}$ and since $\varepsilon>0$ was arbitrary, it follows that $u\left(x_{0}\right) \leq u^{*}\left(x_{0}\right)$. 
On the other hand, $x_{0}$ is a Lebesgue point and, thus,

$$
u^{*}\left(x_{0}\right)=\underset{y \rightarrow x_{0}}{\operatorname{ess} \lim _{n} \inf } u(y) \leq \lim _{R \rightarrow 0} f_{B\left(x_{0}, R\right)} u(x) d \mu=u\left(x_{0}\right) .
$$

Consequently, $u^{*}=u$ q.e. in $\Omega$.

If $u$ is a minimizer, then $u^{*}$ is a continuous minimizer (see Proposition 3.8 and Corollary 5.5 in Kinnunen-Shanmugalingam [36]). A p-harmonic function is a continuous minimizer.

We follow Kinnunen-Martio [33] in giving the following definition of the obstacle problem. Let $V \subset X$ be a nonempty bounded open set with $C_{p}(X \backslash$ $V)>0$. (If $X$ is unbounded then the condition $C_{p}(X \backslash V)>0$ is of course immediately fulfilled.)

Definition 5.3. Let $f \in N^{1, p}(V)$ and $\psi: V \rightarrow \overline{\mathbb{R}}$. Then we define

$$
\mathcal{K}_{\psi, f}(V)=\left\{v \in N^{1, p}(V): v-f \in N_{0}^{1, p}(V) \text { and } v \geq \psi \text { a.e. in } V\right\} .
$$

Further, a function $u \in \mathcal{K}_{\psi, f}(V)$ is a solution of the $\mathcal{K}_{\psi, f}(V)$-obstacle problem if

$$
\int_{V} g_{u}^{p} d \mu \leq \int_{V} g_{v}^{p} d \mu \quad \text { for all } v \in \mathcal{K}_{\psi, f}(V) .
$$

Kinnunen-Martio [33, Theorem 3.2], showed that if $\mathcal{K}_{\psi, f}(V) \neq \varnothing$, then there is a solution of the $\mathcal{K}_{\psi, f}(V)$-obstacle problem, and this solution is unique up to equivalence in $N^{1, p}(V)$. They also showed, Theorem 5.1 in [33], that if $u$ is a solution, then $u^{*}$ is the unique ess lim inf-regularized solution. Furthermore, $u^{*}$ is superharmonic in $V$ (see below). If the obstacle $\psi$ is continuous they showed that $u^{*}$ is also continuous, see Theorem 5.5 in [33]. They actually considered continuous functions which are even allowed to take the value $-\infty$. For $f \in N^{1, p}(V)$, define $H_{V} f$ to be the continuous solution of the $\mathcal{K}_{-\infty, f}(V)$-obstacle problem.

A solution $u$ of the $\mathcal{K}_{\psi, f}(V)$-obstacle problem is a superminimizer in $V$. Conversely, if $u \in N^{1, p}(V)$ is a superminimizer, then $u$ is a solution of the $\mathcal{K}_{u, u}(V)$-obstacle problem.

Definition 5.4. A function $u: \Omega \rightarrow(-\infty, \infty]$ is superharmonic in $\Omega$ if

(i) $u$ is lower semicontinuous;

(ii) $u$ is not identically $\infty$ in any component of $\Omega$;

(iii) for every nonempty open set $V \Subset \Omega$ and all functions $v \in \operatorname{Lip}(X)$, we have $H_{V} v \leq u$ in $V$ whenever $v \leq u$ on $\partial V$.

For us it will be convenient to know that $u$ is superharmonic if and only if $(i i)$ holds and $\min \{u, k\}$ is an ess lim inf-regularized superminimizer for every $k \in \mathbb{R}$, see Theorem 6.1 in A. Björn [1], which also shows that our definition of superharmonic functions is equivalent to the definitions used in Heinonen-Kilpeläinen-Martio [23] and Kinnunen-Martio [33]. 


\section{Lebesgue points for superharmonic functions}

Every locally bounded superharmonic function (or, which is the same, locally bounded ess lim inf-regularized superminimizer) has Lebesgue points everywhere, which was observed by Kinnunen-Martio [33, Remark 5.4]. For unbounded superharmonic functions this is not true, but we can go one step further than Kinnunen and Martio showing that also unbounded superharmonic functions have $L^{q}$-Lebesgue points everywhere for certain $q$. Moreover we show that our range of $q$ is sharp.

We will need the following sharp weak Harnack inequality.

Theorem 6.1. Assume that $X$ supports a $(\varkappa p, p)$-Poincaré inequality, with dilation constant $\lambda$, for some $\varkappa>1$ and that $0<\sigma<\varkappa(p-1)$. Let $u \geq 0$ be superharmonic in $\Omega$, then there is $C>0$, only depending on $p, \varkappa, C_{\mu}$ and the constants in the Poincaré inequality, such that

$$
\left(f_{B} u^{\sigma} d \mu\right)^{1 / \sigma} \leq C \inf _{B} u
$$

for every ball $B \subset 20 \lambda B \Subset \Omega$.

Proof. This follows from Theorem 9.2 in Björn-Marola [9] as in KinnunenMartio [35], Theorems 4.3 and 5.1. Note that the results in [35] need to be modified, taking $\lambda$ into account, see the discussion in Section 10 in [9].

Proposition 6.2. Assume that $u$ is a locally bounded superharmonic function in $\Omega$. Then

$$
\lim _{r \rightarrow 0} f_{B\left(x_{0}, r\right)}\left|u-u\left(x_{0}\right)\right| d \mu=0 \quad \text { for all } x_{0} \in \Omega .
$$

Proof. We may assume that $0<u<1$. Let $0<\varepsilon<u\left(x_{0}\right)$. As $u$ is lower semicontinuous there is $r^{\prime}$ such that $u>u\left(x_{0}\right)-\varepsilon$ in $B\left(x_{0}, r^{\prime}\right)$. Let $v=u-\left(u\left(x_{0}\right)-\varepsilon\right), \sigma=\min \{\varkappa(p-1) / 2,1\}$ and $r<r^{\prime} / 20 \lambda$. Then $0<v<1$ in $B\left(x_{0}, r^{\prime}\right)$ and, by Theorem 6.1 ,

$$
\begin{aligned}
f_{B\left(x_{0}, r\right)}\left|u-u\left(x_{0}\right)\right| d \mu & =f_{B\left(x_{0}, r\right)}\left|v-v\left(x_{0}\right)\right| d \mu \leq v\left(x_{0}\right)+f_{B\left(x_{0}, r\right)} v^{1-\sigma} v^{\sigma} d \mu \\
& \leq \varepsilon+f_{B\left(x_{0}, r\right)} v^{\sigma} d \mu \leq \varepsilon+C \varepsilon^{\sigma} .
\end{aligned}
$$

Letting $r \rightarrow 0$ and then $\varepsilon \rightarrow 0$ completes the proof. 
Proposition 6.3. Assume that $u$ is essentially locally bounded in $\Omega$ and let $x_{0} \in \Omega$. If

$$
\lim _{r \rightarrow 0} f_{B\left(x_{0}, r\right)}\left|u-u\left(x_{0}\right)\right|^{q} d \mu=0
$$

for some $q=q_{0}>0$ then (6.2) holds for all $q>0$.

Note that we do not assume that $u$ is superharmonic, this result holds for any function.

Proof. Assume that (6.2) holds for $q=q_{0}$. If $q<q_{0}$ then it follows from Hölder's inequality that (6.2) holds even for unbounded $u$.

Assume therefore that $q>q_{0}$. We may also assume that $0 \leq u \leq 1$ in $B\left(x_{0}, r^{\prime}\right)$ for some $r^{\prime}>0$. Then, for $0<r<r^{\prime}$ we get that

$$
\begin{aligned}
f_{B\left(x_{0}, r\right)}\left|u-u\left(x_{0}\right)\right|^{q} d \mu & =f_{B\left(x_{0}, r\right)}\left|u-u\left(x_{0}\right)\right|^{q-q_{0}}\left|u-u\left(x_{0}\right)\right|^{q_{0}} d \mu \\
& \leq f_{B\left(x_{0}, r\right)}\left|u-u\left(x_{0}\right)\right|^{q_{0}} d \mu \rightarrow 0, \quad \text { as } r \rightarrow 0 .
\end{aligned}
$$

Theorem 6.4. Assume that $u$ is a superharmonic function in $\Omega$. Let $\sigma$ be as in Theorem 6.1. Then

$$
\lim _{r \rightarrow 0} f_{B\left(x_{0}, r\right)}\left|u-u\left(x_{0}\right)\right|^{\sigma} d \mu=0, \quad \text { if } u\left(x_{0}\right)<\infty,
$$

and, for every $q>0$,

$$
\lim _{r \rightarrow 0} f_{B\left(x_{0}, r\right)} u^{q} d \mu=\infty, \quad \text { if } u\left(x_{0}\right)=\infty .
$$

Proof. When $u\left(x_{0}\right)<\infty$, the proof is fairly similar to the proof of Proposition 6.2. Indeed, let $\varepsilon>0$. As $u$ is lower semicontinuous there is $r^{\prime}$ such that $u>u\left(x_{0}\right)-\varepsilon$ in $B\left(x_{0}, r^{\prime}\right)$. Let $v=u-\left(u\left(x_{0}\right)-\varepsilon\right)$ and $r<r^{\prime} / 20 \lambda$. Then, by Theorem 6.1 ,

$$
\begin{aligned}
f_{B\left(x_{0}, r\right)}\left|u-u\left(x_{0}\right)\right|^{\sigma} d \mu & =f_{B\left(x_{0}, r\right)}\left|v-v\left(x_{0}\right)\right|^{\sigma} d \mu \leq v\left(x_{0}\right)^{\sigma}+f_{B\left(x_{0}, r\right)} v^{\sigma} d \mu \\
& \leq v\left(x_{0}\right)^{\sigma}+C v\left(x_{0}\right)^{\sigma} \leq \varepsilon^{\sigma}+C \varepsilon^{\sigma} .
\end{aligned}
$$

Letting $\varepsilon \rightarrow 0$ completes the proof of (6.3).

If $u\left(x_{0}\right)=\infty$ we instead proceed as follows. Let $\omega>0$. As $u$ is lower semicontinuous there is $r^{\prime}$ such that $u>\omega$ in $B\left(x_{0}, r^{\prime}\right)$. It follows that

$$
\lim _{r \rightarrow 0} f_{B\left(x_{0}, r\right)} u^{q} d \mu \geq \omega^{q} .
$$

Letting $\omega \rightarrow \infty$ completes the proof of (6.4). 
Remark 6.5. Let us demonstrate the sharpness of Theorem 6.4. In unweighted $\mathbb{R}^{n}$ with $2 \leq p<n$, we have $s=n$ in (4.1) and $\mathbb{R}^{n}$ supports a $\left(p^{*}, p\right)$-Poincaré inequality, where $p^{*}=n p /(n-p)$. This is proved e.g. in Theorem 2, p. 141, in Evans-Gariepy [16] as well as in Corollary 1.64 in MalýZiemer [40] (and for metric spaces in Theorem 5.1 in Hajłasz-Koskela [18]). We can thus have any positive $\sigma<\sigma^{*}:=p^{*}(p-1) / p=n(p-1) /(n-p)$ in Theorems 6.1 and 6.4 .

$$
\begin{aligned}
& \text { Let } v(x)=|x|^{(p-n) /(p-1)}, x_{j}=\left(2^{-j}, 0, \ldots, 0\right) \\
& \qquad u_{N}(x)=\sum_{j=1}^{N} 2^{-j} \frac{v\left(x+x_{j}\right)}{v\left(x_{j}\right)} \text { and } u(x)=\sum_{j=1}^{\infty} 2^{-j} \frac{v\left(x+x_{j}\right)}{v\left(x_{j}\right)} .
\end{aligned}
$$

By Theorem 3.1 in Crandall-Zhang [14] (here we use that $p \geq 2$ ) and Theorem 7.35 in Heinonen-Kilpeläinen-Martio [23], $u_{N}$ is superharmonic in $\mathbb{R}^{n}$. It follows from Lemma 7.3 in [23] (or Theorem 8.2) that $u=\lim _{N \rightarrow \infty} u_{N}$ is superharmonic in $\mathbb{R}^{n}$. As $u(0)=1$ and

$$
f_{B(0, r)} u^{\sigma^{*}} d \mu=\infty
$$

we see that (6.3) fails for $\sigma=\sigma^{*}$.

Proposition 6.6. Assume that $u$ is a superharmonic function in $\Omega$. Let $\sigma$ be as in Theorem 6.1. Then

$$
u\left(x_{0}\right)=\lim _{r \rightarrow 0}\left(f_{B\left(x_{0}, r\right)} u^{\sigma} d \mu\right)^{1 / \sigma} \quad \text { for all } x_{0} \in \Omega,
$$

where we interpret $u^{\sigma}$ as $|u|^{\sigma}$ sign $u$, and similarly for other powers.

Let further $q>0$ and $u_{k}:=\min \{u, k\}$. Then

$$
u\left(x_{0}\right)=\lim _{k \rightarrow \infty} \lim _{r \rightarrow 0}\left(f_{B\left(x_{0}, r\right)} u_{k}^{q} d \mu\right)^{1 / q} \text { for all } x_{0} \in \Omega .
$$

In weighted $\mathbb{R}^{n}$ the first part was obtained for $\sigma=1$ and ess lim infregularized superminimizers $u$, whenever $\sigma=1$ is permitted, in Theorem 3.66 in Heinonen-Kilpeläinen-Martio [23].

Proof. The first part follows directly from (6.4) when $u\left(x_{0}\right)=\infty$. When $u\left(x_{0}\right)<\infty$ and $\sigma \geq 1$, it follows from (6.3) by means of the triangle (Minkowski) inequality. For $\sigma<1$ we need to use the elementary inequality

$$
\left|a^{\sigma}-b^{\sigma}\right| \leq 2^{1-\sigma}|a-b|^{\sigma} .
$$


In the second part we use (6.5) together with Propositions 6.2 and 6.3 to get that

$$
u_{k}\left(x_{0}\right)=\lim _{r \rightarrow 0}\left(f_{B\left(x_{0}, r\right)} u_{k}^{q} d \mu\right)^{1 / q} \quad \text { for all } x_{0} \in \Omega .
$$

From which the last part follows directly.

\section{Convergence of superminimizers}

In this section, we prove that the limit of a decreasing sequence of superminimizers is a superminimizer provided, of course, that there exists a suitable lower bound. Observe also that then the ess lim inf-regularization changes the limit only in a set of capacity zero. This result provides a straightforward proof of the fundamental convergence theorem of potential theory as we shall show in Section 8. By combining the decreasing convergence result with the increasing convergence result (Theorem 7.3), we obtain convergence for nonmonotone sequences in Corollary 7.5 as well.

Theorem 7.1. Let $\left\{u_{i}\right\}_{i=1}^{\infty}$ be a decreasing sequence of superminimizers in $\Omega$ such that $u_{i} \geq f$ a.e. in $\Omega$ for some $f \in N_{\text {loc }}^{1, p}(\Omega)$. Let $u=\lim _{i \rightarrow \infty} u_{i}$. Then

(a) $u$ is a superminimizer in $\Omega$;

(b) $u^{*}$ is superharmonic in $\Omega$;

(c) $u=u^{*}$ q.e. in $\Omega$.

We provide two proofs of Theorem 7.1. Part (b) is quite straightforward to deduce directly, as we show in the second proof. The difficult part is to obtain (c). In both proofs we first deduce that $u \in N_{\text {loc }}^{1, p}(\Omega)$.

In the first proof we proceed to show (a), after which (c) follows directly from Theorem 5.2 and (b) from Proposition 7.4 in Kinnunen-Martio [33] (and we do not need the direct deduction of (b) mentioned above).

Alternatively we proceed as in the second proof, where we first prove (c) using ideas similar to our proof of Theorem 5.2, mainly the useful robustness of the supremum estimate (5.3) for decreasing sequences. In this proof we use the direct deduction of (b) (which does not come for free as it did in the first proof). After this (a) follows in a straightforward manner from results in [33].

Our result has one important difference to the existing versions in the Euclidean literature: superminimizers in $\mathbb{R}^{n}$ belong to the usual Sobolev spaces with a.e.-equivalence classes, which makes it impossible to deduce (c) directly from (a). Also (c) is known in the Euclidean setting, see HeinonenKilpeläinen [21] or Theorem 8.2 in Heinonen-Kilpeläinen-Martio [23], but 
the proof uses advanced tools from potential theory. Both our proofs use direct methods in the calculus of variations in a straightforward manner.

Lemma 7.2. Let $\left\{u_{i}\right\}_{i=1}^{\infty}$ be a sequence of superminimizers in $\Omega$ such that $u_{i} \rightarrow u$ q.e. in $\Omega$. If there is a function $f \in N_{\mathrm{loc}}^{1, p}(\Omega)$ such that $\left|u_{i}\right| \leq f$ a.e. in $\Omega, i=1,2, \ldots$, then $u \in N_{\text {loc }}^{1, p}(\Omega)$.

In particular, Lemma 7.2 applies if the sequence $\left\{u_{i}\right\}_{i=1}^{\infty}$ is decreasing (increasing) and $u$ is locally essentially bounded from below (above).

Proof. Clearly, $u \in L_{\text {loc }}^{p}(\Omega)$. If we can show that $\left\{g_{u_{i}}\right\}_{i=1}^{\infty}$ is uniformly bounded in $L^{p}(B)$ for every $B \Subset \Omega$, then we can deduce that $u \in N_{\text {loc }}^{1, p}(\Omega)$ using Corollary 3.3.

To this end, let $B=B\left(x_{0}, R\right) \Subset \Omega$. Then we can find $R^{\prime}>R$ so that also $B^{\prime}=B\left(x_{0}, R^{\prime}\right) \Subset \Omega$. Let next $0<r_{1}<r_{2} \leq R^{\prime}, B_{j}=B\left(x_{0}, r_{j}\right), j=1,2$, and

$$
\eta=\min \left\{\frac{r_{2}-d\left(x_{0}, x\right)}{r_{2}-r_{1}}, 1\right\}_{+} \in N_{0}^{1, p}(B) .
$$

We will use that

$$
g_{\eta} \leq \frac{1}{r_{2}-r_{1}} \chi_{B_{2} \backslash B_{1}} .
$$

Set $\varphi_{i}=\eta\left(f-u_{i}\right) \in N_{0}^{1, p}\left(B_{2}\right)$, which is nonnegative a.e. in $\Omega$. As $\left(\varphi_{i}\right)_{-}=0$ a.e. in $X$ and $\left(\varphi_{i}\right)_{-} \in N^{1, p}(X)$, it follows that $\left(\varphi_{i}\right)_{-}=0$ q.e. in $\Omega$, and hence that $\varphi_{i} \geq 0$ q.e. in $\Omega$. Since $\varphi_{i}$ and $\left(\varphi_{i}\right)_{+}$are representatives of the same equivalence class in $N_{0}^{1, p}\left(B_{2}\right)$, we can assume that $\varphi_{i}$ is nonnegative everywhere in $\Omega$. By Lemma 2.4 in Kinnunen-Martio [33], we have that

$$
g_{u_{i}+\varphi_{i}} \leq(1-\eta) g_{u_{i}}+\left(f-u_{i}\right) g_{\eta}+\eta g_{f} \quad \text { a.e. in } B \text {. }
$$

Since $u_{i}$ is a superminimizer we have that

$$
\begin{aligned}
\int_{B_{1}} g_{u_{i}}^{p} d \mu & \leq \int_{B_{2}} g_{u_{i}}^{p} d \mu \leq \int_{B_{2}} g_{u_{i}+\varphi_{i}}^{p} d \mu \\
& \leq 3^{p}\left(\int_{B_{2}}(1-\eta)^{p} g_{u_{i}}^{p} d \mu+\int_{B_{2}}\left(f-u_{i}\right)^{p} g_{\eta}^{p} d \mu+\int_{B_{2}} \eta^{p} g_{f}^{p} d \mu\right) \\
& \leq 3^{p}\left(\int_{B_{2} \backslash B_{1}} g_{u_{i}}^{p} d \mu+\frac{2^{p}}{\left(r_{2}-r_{1}\right)^{p}} \int_{B^{\prime}} f^{p} d \mu+\int_{B^{\prime}} g_{f}^{p} d \mu\right) .
\end{aligned}
$$

Adding $3^{p}$ times the left-hand side to both sides we obtain that

$$
\left(1+3^{p}\right) \int_{B_{1}} g_{u_{i}}^{p} d \mu \leq 3^{p}\left(\int_{B_{2}} g_{u_{i}}^{p} d \mu+\frac{2^{p}}{\left(r_{2}-r_{1}\right)^{p}} \int_{B^{\prime}} f^{p} d \mu+\int_{B^{\prime}} g_{f}^{p} d \mu\right) .
$$


After dividing by $1+3^{p}$ we get, with $\theta=3^{p} /\left(1+3^{p}\right)<1$, that

$$
\int_{B_{1}} g_{u_{i}}^{p} d \mu \leq \theta \int_{B_{2}} g_{u_{i}}^{p} d \mu+\frac{2^{p}}{\left(r_{2}-r_{1}\right)^{p}} \int_{B^{\prime}} f^{p} d \mu+\int_{B^{\prime}} g_{f}^{p} d \mu
$$

By Lemma 3.1, p. 161, in Giaquinta [17], we have that

$$
\int_{B_{1}} g_{u_{i}}^{p} d \mu \leq C\left(\frac{1}{\left(r_{2}-r_{1}\right)^{p}} \int_{B^{\prime}} f^{p} d \mu+\int_{B^{\prime}} g_{f}^{p} d \mu\right)
$$

for $0 \leq r_{1}<r_{2} \leq R^{\prime}$. By choosing $r_{1}=R$ we see that $\left\{g_{u_{i}}\right\}_{i=1}^{\infty}$ is bounded in $L^{p}(B)$. By Corollary 3.3, $u \in N^{1, p}(B)$, and hence $u \in N_{\text {loc }}^{1, p}(\Omega)$.

The next theorem generalizes Remark 6.7(1) in Kinnunen-Martio [33] and gives a necessary and sufficient condition for when an increasing limit of superminimizers is a superminimizer. It was pointed out by T. Ono [41].

Theorem 7.3. Let $\left\{u_{i}\right\}_{i=1}^{\infty}$ be an increasing sequence of superminimizers in $\Omega$ and assume that there is a function $f \in N_{\mathrm{loc}}^{1, p}(\Omega)$ such that $u:=$ $\lim _{i \rightarrow \infty} u_{i} \leq f$ a.e. in $\Omega$. Then $u$ is a superminimizer in $\Omega$, $u^{*}$ is superharmonic in $\Omega$ and $u=u^{*}$ q.e. in $\Omega$.

Note that if all $u_{i}$ are ess liminf-regularized then so is $u$. This is not completely trivial but follows from Lemma 7.1 in Kinnunen-Martio [33].

Proof. Since $\left|u_{i}\right| \leq \max \left\{\left|u_{1}\right|, f\right\}$ a.e. in $\Omega$ and $\max \left\{\left|u_{1}\right|, f\right\} \in N_{\text {loc }}^{1, p}(\Omega)$, Lemma 7.2 shows that $u \in N_{\text {loc }}^{1, p}(\Omega)$. The result now follows from Remark 6.7(1) and Proposition 7.4 in [33], together with Theorem 5.2.

As a consequence we obtain the following result.

Proposition 7.4. If $u$ is a superharmonic function in $\Omega$ which is bounded from above by an $N_{\text {loc }}^{1, p}(\Omega)$-function, then $u$ is a superminimizer.

Proof. We know that $u_{k}:=\min \{u, k\}$ is a superminimizer, $k=1,2, \ldots$, see the comments after Definition 5.4. Thus, by Theorem 7.3, $u=\lim _{k \rightarrow \infty} u_{k}$ is a superminimizer.

First proof of Theorem 7.1. (a) Since $u_{i} \leq u_{1} \in N_{\text {loc }}^{1, p}(\Omega)$, Lemma 7.2 shows that $u \in N_{\text {loc }}^{1, p}(\Omega)$. Let $G \Subset \Omega$ be open and $v$ be a solution of the $\mathcal{K}_{u, u}(G)$-obstacle problem. As $u_{i}$ is a solution of the $\mathcal{K}_{u_{i}, u_{i}}(G)$-obstacle problem, Lemma 5.4 in Björn-Björn [4] implies that $v \leq u_{i}$ q.e. in $G$. Furthermore, as this holds for all $i$ we have $v \leq u$ q.e. in $G$.

On the other hand, by the definition of the obstacle problem, $v \geq u$ a.e. in $G$, and thus $u=v$ a.e. in $G$. As $u, v \in N^{1, p}(G)$, we get that $u=v$ q.e. in 
$G$, and thus $u$ is also a solution of the $\mathcal{K}_{u, u}(G)$-obstacle problem. Hence $u$ is a superminimizer in $\Omega$.

(c) This now follows directly by Theorem 5.2.

(b) This now follows from Proposition 7.4 in Kinnunen-Martio [33].

Second proof of Theorem 7.1. Since $u_{i} \leq u_{1} \in N_{\text {loc }}^{1, p}(\Omega)$, Lemma 7.2 shows that $u \in N_{\text {loc }}^{1, p}(\Omega)$.

(c) In view of Theorem 4.1, the set

$$
E=\left\{x_{0} \in \Omega: \lim _{R \rightarrow 0} f_{B\left(x_{0}, R\right)}\left|u\left(x_{0}\right)-u\right| d \mu=0 \text { and }\left|u\left(x_{0}\right)\right|<\infty\right\}
$$

differs from $\Omega$ only in a set of capacity zero.

Choose $x_{0} \in E$ and observe that $\left(u\left(x_{0}\right)-u_{i}\right)_{+}$is a positive subminimizer. As in the proof of Theorem 5.2, we have for $B\left(x_{0}, R\right) \Subset \Omega$ that

$$
\operatorname{ess~sup}_{B\left(x_{0}, R / 2\right)}\left(u\left(x_{0}\right)-u_{i}\right)_{+} \leq C f_{B\left(x_{0}, R\right)}\left(u\left(x_{0}\right)-u_{i}\right)_{+} d \mu .
$$

Since the sequence of subminimizers is increasing, we deduce that

$$
\lim _{i \rightarrow \infty} \operatorname{ess~sup}_{B\left(x_{0}, R / 2\right)}\left(u\left(x_{0}\right)-u_{i}\right)_{+}=\operatorname{ess~sup}_{B\left(x_{0}, R / 2\right)}\left(u\left(x_{0}\right)-u\right)_{+} .
$$

By this fact and Lebesgue's monotone convergence theorem, we can then pass to the limit and infer that

$$
\operatorname{ess}_{B\left(x_{0}, R / 2\right)}\left(u\left(x_{0}\right)-u\right)_{+} \leq C f_{B\left(x_{0}, R\right)}\left(u\left(x_{0}\right)-u\right)_{+} d \mu,
$$

i.e. (5.3) holds. The rest of the proof of (c) is similar to the proof of Theorem 5.2 .

(b) Clearly, $u^{*}$ is lower semicontinuous and is not identically $\infty$ in any component. It remains to show that $u^{*}$ satisfies the comparison principle (iii) in Definition 5.4. To this end, let $\Omega^{\prime} \Subset \Omega$ be open and $v \in \operatorname{Lip}(X)$ be such that $v \leq u^{*}$ on $\partial \Omega^{\prime}$. As $u^{*} \leq u_{i}^{*}$, we also have $v \leq u_{i}^{*}$ on $\partial \Omega^{\prime}$. Since $u_{i}^{*}$ is superharmonic it follows that

$$
H_{\Omega^{\prime}} v \leq u_{i}^{*} \text { in } \Omega^{\prime}
$$

Although $u_{i} \rightarrow u$ does not in general imply that $u_{i}^{*} \rightarrow u^{*}$, it follows that $u_{i}^{*} \rightarrow u$ q.e. due to Theorem 5.2. Passing to the limit in (7.1) we obtain that

$$
H_{\Omega^{\prime}} v \leq u \text { q.e. in } \Omega^{\prime}
$$


Since $H_{\Omega^{\prime}} v$ is continuous, we finally end up with

$$
H_{\Omega^{\prime}} v=\left(H_{\Omega^{\prime}} v\right)^{*} \leq u^{*} \quad \text { everywhere in } \Omega^{\prime} .
$$

We have thus shown that $u^{*}$ is superharmonic in $\Omega$.

(a) By (b), $u^{*}$ is superharmonic. As it belongs to $N_{\text {loc }}^{1, p}(\Omega)$, it is also a superminimizer by Proposition 7.4. As $u=u^{*}$ q.e. it follows that also $u$ is a superminimizer in $\Omega$.

Next we prove a convergence result for any pointwise convergent sequence of superminimizers, not necessarily monotone, which is bounded from above and from below by functions in $N_{\text {loc }}^{1, p}(\Omega)$. In particular this is true if the sequence is locally uniformly essentially bounded from below and the limiting function is locally essentially bounded from above. The proof is based on the combination of the increasing and decreasing convergence results.

Corollary 7.5. Let $\left\{u_{i}\right\}_{i=1}^{\infty}$ be a sequence of superminimizers and let

$$
v=\liminf _{i \rightarrow \infty} u_{i} .
$$

If there exist $f_{1}, f_{2} \in N_{\text {loc }}^{1, p}(\Omega)$ such that $u_{i} \geq f_{1}$ a.e. in $\Omega, i=1,2, \ldots$, and $v \leq f_{2}$ a.e. in $\Omega$, then $v$ is a superminimizer in $\Omega, v^{*}$ is superharmonic in $\Omega$ and $v^{*}=v$ q.e. in $\Omega$.

Proof. For every $k=1,2 \ldots$, the functions

$$
v_{k, i}=\min \left\{u_{k}, \ldots, u_{i}\right\}, \quad i \geq k,
$$

are superminimizers and $u_{k} \geq v_{k, i} \geq f_{1}$ a.e. in $\Omega$. Theorem 7.1 implies that $v_{k}=\lim _{i \rightarrow \infty} v_{k, i}$ is also a superminimizer in $\Omega^{\prime}$. The sequence $\left\{v_{k}\right\}_{k=1}^{\infty}$ is increasing and by Theorem 7.3,v $=\lim _{k \rightarrow \infty} v_{k}$ is a superminimizer, $v^{*}$ is superharmonic and $v=v^{*}$ q.e. in $\Omega$.

\section{Convergence of superharmonic functions}

In this section, we extend the convergence results from Section 7 to superharmonic functions and prove the fundamental convergence theorem of potential theory. In the nonlinear Euclidean theory, the proof of the fundamental convergence theorem uses advanced tools from potential theory as we pointed out after Theorem 7.1. Our approach is based on the following convergence theorem for a decreasing sequence of superharmonic functions.

Theorem 8.1. Let $\left\{u_{j}\right\}_{j=1}^{\infty}$ be a decreasing sequence of superharmonic functions in $\Omega$ such that $v:=\lim _{j \rightarrow \infty} u_{j} \geq f$ for some $f \in N_{\text {loc }}^{1, p}(\Omega)$. Then $v^{*}$ is superharmonic in $\Omega$ and $v=v^{*}$ q.e. in $\Omega$. 
Theorem 8.1 is a special case of the following more general result and the proof is virtually the same.

Theorem 8.2. Let $\left\{u_{j}\right\}_{j=1}^{\infty}$ be a sequence of superharmonic functions in $\Omega$ such that $u_{j} \geq f$ a.e. in $\Omega$ for some $f \in N_{\mathrm{loc}}^{1, p}(\Omega)$ and all $j=1,2, \ldots$. Let $v=\liminf _{j \rightarrow \infty} u_{j}$. If $v^{*}$ is not identically $\infty$ in any component of $\Omega$, then it is superharmonic in $\Omega$ and $v=v^{*}$ q.e. in $\Omega$.

Proof. Let $v_{k}:=\min \{v, k\}=\liminf _{j \rightarrow \infty} \min \left\{u_{j}, k\right\}$. Each $\min \left\{u_{j}, k\right\}$ is a superminimizer in $\Omega, \min \left\{u_{j}, k\right\} \geq \min \{f, k\}$ a.e. in $\Omega$ and $v_{k} \leq k \in$ $N_{\text {loc }}^{1, p}(\Omega)$. Corollary 7.5 then implies that $v_{k}$ is a superminimizer in $\Omega, v_{k}=v_{k}^{*}$ q.e. in $\Omega$ and $v_{k}^{*}$ is superharmonic in $\Omega$.

As $v_{k}^{*}=\min \left\{v^{*}, k\right\}$ in $\Omega$, the characterization after Definition 5.4 shows that $v^{*}$ is superharmonic in $\Omega$. Moreover, $v^{*}=\lim _{k \rightarrow \infty} v_{k}^{*}=\lim _{k \rightarrow \infty} v_{k}=v$ q.e. in $\Omega$.

The main aim in this section is to prove the following result. First we need to define the liminf-regularization of a function $f: \Omega \rightarrow \overline{\mathbb{R}}$ as

$$
\hat{f}(x)=\lim _{r \rightarrow 0} \inf _{\Omega \cap B(x, r)} f, \quad x \in \Omega .
$$

It follows that $\hat{f} \leq f$, and it is easy to show that $\hat{f}$ is lower semicontinuous.

Theorem 8.3. (The fundamental convergence theorem) Let $\mathcal{F}$ be a nonempty family of superharmonic functions in $\Omega$. Assume that there is $f \in$ $N_{\text {loc }}^{1, p}(\Omega)$ such that $u \geq f$ a.e. in $\Omega$ for all $u \in \mathcal{F}$. Let $w=\inf \mathcal{F}$. Then the following are true:

(a) $\widehat{w}$ is superharmonic;

(b) $\widehat{w}=w^{*}$ in $\Omega$;

(c) $\widehat{w}=w$ q.e. in $\Omega$.

Proving (b) is straightforward, as we will show below. Also (a) can be obtained directly in the same way as in Lemma 7.4 in Heinonen-KilpeläinenMartio [23]. The difficult part is to prove (c). Our proof of (c) is based on Theorem 8.1 and automatically gives (a).

Usually, in the fundamental convergence theorem it is assumed that the functions in $\mathcal{F}$ are locally uniformly bounded, rather than the (slightly) more general condition here. In fact, it follows from our result that under our condition the functions in $\mathcal{F}$ are locally uniformly bounded. The advantage with our formulation is that it allows for connecting balayage and obstacle problems without requiring an unnatural (and unnecessary) condition on which obstacle problems are under consideration. This connection is established in Björn-Björn-Mäkäläinen-Parviainen [5]. In [5] the fundamental convergence theorem is used as a starting point for the development 
of the theory of balayage, this is in contrast to earlier developments of the theory of balayage where the fundamental convergence theorem is obtained as a consequence of the theory.

We will need Choquet's topological lemma. We say that a family of functions $\mathcal{U}$ is downward directed if for each $u, v \in \mathcal{U}$ there is $w \in \mathcal{U}$ with $w \leq \min \{u, v\}$.

Lemma 8.4. (Choquet's topological lemma) Let $\mathcal{U}=\left\{u_{\gamma}: \gamma \in I\right\}$ be a nonempty family of functions $u_{\gamma}: \Omega \rightarrow \overline{\mathbb{R}}$. Let $u=\inf \mathcal{U}$. If $\mathcal{U}$ is downward directed, then there is a decreasing sequence of functions $v_{j} \in \mathcal{U}$ with $v=\lim _{j \rightarrow \infty} v_{j}$ such that $\hat{v}=\hat{u}$.

Proof. The proof of Lemma 8.3 in Heinonen-Kilpeläinen-Martio [23] generalizes directly to metric spaces. Just remember that our metric space $X$ is separable.

Proof of Theorem 8.3. (b) It is clear that $w^{*} \geq \widehat{w}$.

For the converse inequality let $B=B(x, r) \subset \Omega$ be a ball and $\varepsilon>0$. If $\widehat{w}(x)=\infty$, then $w^{*}(x) \leq \widehat{w}(x)$ trivially, so we may assume that $\widehat{w}(x)<\infty$. We can then find $y \in B$ such that $w(y)<\widehat{w}(x)+\varepsilon$ and hence also $u \in \mathcal{F}$ such that $u(y)<\widehat{w}(x)+\varepsilon$. As $u$ is superharmonic and hence ess liminfregularized, it follows that

$$
\mu(\{z \in B: w(z)<\widehat{w}(x)+\varepsilon\}) \geq \mu(\{z \in B: u(z)<\widehat{w}(x)+\varepsilon\})>0 .
$$

Since this is true for all balls $B=B(x, r) \subset \Omega$ we see that $w^{*}(x) \leq \widehat{w}(x)+\varepsilon$. Letting $\varepsilon \rightarrow 0$ shows that $w^{*} \leq \widehat{w}$ and thus $w^{*}=\widehat{w}$. Then

(a) and (c) Let $\mathcal{U}=\{u: u$ is superharmonic in $\Omega$ and $u \geq w$ in $\Omega\} \supset \mathcal{F}$.

$$
w \leq \inf _{u \in \mathcal{U}} u \leq \inf _{u \in \mathcal{F}} u=w .
$$

As $\mathcal{U}$ is downward directed, by Choquet's topological lemma (Lemma 8.4) there is a decreasing sequence of superharmonic functions $v_{j} \in \mathcal{U}$ with $v=$ $\lim _{j \rightarrow \infty} v_{j}$ such that $\hat{v}=\widehat{w}$. By (b) applied to $v$ we have that $\widehat{w}=\hat{v}=v^{*}$ everywhere in $\Omega$. Theorem 8.1 applied to the sequence $\left\{v_{j}\right\}_{j=1}^{\infty}$ shows that $v^{*}$ is superharmonic and $v^{*}=v$ q.e. in $\Omega$. Finally, as $v_{j} \geq w, j=1,2, \ldots$, we get that

$$
\widehat{w}=v^{*}=v \geq w \geq \widehat{w} \text { q.e. in } \Omega .
$$

Example 8.5. The lower semicontinuous regularization is necessary in the fundamental convergence theorem. To see this consider the sequence of superharmonic functions $u_{j}(x)=|x|^{(p-n) /(n-1)} / j, j=1,2, \ldots$, (with $u_{j}(0)=$ $\infty)$ in unweighted $\mathbb{R}^{n}$ with $1<p<n$. The infimum is clearly $u(x)=0$ for $x \neq 0$ and $u(0)=\infty$, which is not lower semicontinuous and hence not superharmonic. The regularization of $u$ is identically zero. 


\section{References}

[1] BJÖRN, A.: Characterizations of $p$-superharmonic functions on metric spaces. Studia Math. 169 (2005), no. 1, 45-62.

[2] BJörn, A.: A weak Kellogg property for quasiminimizers. Comment. Math. Helv. 81 (2006), no. 4, 809-825.

[3] BJöRN, A.: A regularity classification of boundary points for $p$-harmonic functions and quasiminimizers. J. Math. Anal. Appl. 338 (2008), 39-47.

[4] BJÖRn, A. AND BJÖRN, J.: Boundary regularity for $p$-harmonic functions and solutions of the obstacle problem. J. Math. Soc. Japan 58 (2006), no. 4, 1211-1232.

[5] Buörn, A., Buörn, J., MÄKÄLÄinen, T. And Parviainen, M.: Nonlinear balayage on metric spaces. Nonlinear Anal. 71 (2009), no. 5-6, 2153-2171.

[6] Björn, A., Buörn, J. And Shanmugalingam, N.: The Dirichlet problem for $p$-harmonic functions on metric spaces. J. Reine Angew. Math. 556 (2003) 173-203.

[7] Björn, A., Buörn, J. and Shanmugalingam, N.: The Perron method for p-harmonic functions. J. Differential Equations 195 (2003), 398-429.

[8] Björn, A., BJörn, J. And Shanmugalingam, N.: Quasicontinuity of Newton-Sobolev functions and density of Lipschitz functions on metric spaces. Houston J. Math. 34 (2008), no. 4, 1197-1211.

[9] Björn, A. ANd Marola, N.: Moser iteration for (quasi)minimizers on metric spaces. Manuscripta Math. 121 (2006), no. 3, 339-366.

[10] BJöRn, J.: Boundary continuity for quasiminimizers on metric spaces. Illinois J. Math. 46 (2002), no. 2, 383-403.

[11] BJöRN, J.: Fine continuity on metric spaces. Manuscripta Math. 125 (2008), no. 3, 369-381.

[12] Buörn, J., Macmanus, P. and Shanmugalingam, N.: Fat sets and pointwise boundary estimates for $p$-harmonic functions in metric spaces. J. Anal. Math. 85 (2001), 339-369.

[13] Cheeger, J.: Differentiability of Lipschitz functions on metric measure spaces. Geom. Funct. Anal. 9 (1999), no. 3, 428-517.

[14] Crandall, M. G. and Zhang, J. Another way to say harmonic. Trans. Amer. Math. Soc. 355 (2003), no. 1, 241-263.

[15] Doob, J. L.: Classical potential theory and its probabilistic counterpart. Springer-Verlag, New York, 1984.

[16] Evans, L. C. And Gariepy, R. F.: Measure theory and fine properties of functions. Studies in Advanced Math. CRC Press, Boca Raton, FL, 1992.

[17] Giaquinta, M.: Multiple integrals in the calculus of variations and nonlinear elliptic systems. Annals of Mathematics Studies 105. Princeton University Press, Princeton, NJ, 1983. 
[18] HajŁasz, P. and Koskela, P.: Sobolev met Poincaré. Mem. Amer. Math. Soc. 145 (2000), no. 688.

[19] Heinonen, J.: Lectures on analysis on metric spaces. Universitext. Springer-Verlag, New York, 2001.

[20] Heinonen, J.: Nonsmooth calculus. Bull. Amer. Math. Soc. 44 (2007), no. $2,163-232$.

[21] Heinonen, J. And Kilpeläinen, T.: Polar sets for supersolutions of degenerate elliptic equations. Math. Scand. 63 (1988), no. 1, 136-150.

[22] Heinonen, J. And Kilpeläinen, T.: On the Wiener criterion and quasilinear obstacle problems. Trans. Amer. Math. Soc. 310 (1988), 239-255.

[23] Heinonen, J., Kilpeläinen, T. and Martio, O.: Nonlinear potential theory of degenerate elliptic equations. Dover Pubs., Mineola, NY, 2006.

[24] Heinonen, J. And Koskela, P.: Quasiconformal maps in metric spaces with controlled geometry. Acta Math. 181 (1998), no. 1, 1-61

[25] Kallunki, S. and Shanmugalingam, N.: Modulus and continuous capacity. Ann. Acad. Sci. Fenn. Math. 26 (2001), no. 2, 455-464.

[26] Keith, S. And Zhong, X.: The Poincaré inequality is an open ended condition. Ann. of Math. (2) 167 (2008), no. 2, 575-599.

[27] KilpeläInen, T.: Potential theory for supersolutions of degenerate elliptic equations. Indiana Univ. Math. J. 38 (1989), no. 2, 253-275.

[28] Kilpeläinen, T.: Weighted Sobolev spaces and capacity. Ann. Acad. Sci. Fenn. Ser. A I Math. 19 (1994), no. 1, 95-113.

[29] Kilpeläinen, T., Kinnunen, J. and Martio, O.: Sobolev spaces with zero boundary values on metric spaces. Potential Anal. 12 (2000), 233-247.

[30] Kinnunen, J. and Latvala, V.: Lebesgue points for Sobolev functions on metric spaces. Rev. Mat. Iberoamericana 18 (2002), no. 3, 685-700.

[31] Kinnunen, J. and Martio, O.: The Sobolev capacity on metric spaces. Ann. Acad. Sci. Fenn. Math. 21 (1996), no. 2, 367-382.

[32] Kinnunen, J. and Martio, O.: Choquet property for the Sobolev capacity in metric spaces. In Proceedings on Analysis and Geometry (Novosibirsk, Akademgorodok, 1999), 285-290. Sobolev Institute Press, Novosibirsk, 2000.

[33] Kinnunen, J. and Martio, O.: Nonlinear potential theory on metric spaces. Illinois J. Math. 46 (2002), no. 3, 857-883.

[34] Kinnunen, J. and Martio, O.: Potential theory of quasiminimizers. Ann. Acad. Sci. Fenn. Math. 28 (2003), no. 2, 459-490.

[35] Kinnunen, J. and Martio, O.: Sobolev space properties of superharmonic functions on metric spaces. Results Math. 44 (2003), 114-129.

[36] Kinnunen, J. and Shanmugalingam, N.: Regularity of quasi-minimizers on metric spaces. Manuscripta Math. 105 (2001), no. 3, 401-423.

[37] Kinnunen, J. And Shanmugalingam, N.: Polar sets on metric spaces. Trans. Amer. Math. Soc. 358 (2006), no. 1, 11-37. 
[38] Koskela, P. And MacManus, P.: Quasiconformal mappings and Sobolev spaces. Studia Math. 131 (1998), no. 1, 1-17.

[39] KuUsi, T.: Lower semicontinuity of weak supersolutions to a nonlinear parabolic equation. Differential Integral Equations 22 (2009), 1211-1222.

[40] Malý, J. And Ziemer, W. P.: Fine regularity of solutions of elliptic partial differential equations. Mathematical Surveys and Monographs 51. Amererican Mathematical Society, Providence, RI, 1997.

[41] Ono, T.: Private communication, 2004.

[42] Shanmugalingam, N.: Newtonian spaces: An extension of Sobolev spaces to metric measure spaces. Rev. Mat. Iberoamericana 16 (2000), 243-279.

[43] Shanmugalingam, N.: Harmonic functions on metric spaces. Illinois J. Math. 45 (2001), no. 3, 1021-1050.

[44] Shanmugalingam, N.: Some convergence results for $p$-harmonic functions on metric measure spaces. Proc. London Math. Soc. (3) 87 (2003), no. 1, 226-246.

[45] Yosida, K.: Functional analysis. Grundlehren der Mathematischen Wissenschaften 123. Springer-Verlag, Berlin-New York, 1980.

Recibido: 28 de mayo de 2008

Revisado: 19 de agosto de 2008

Anders Björn

Department of Mathematics

Linköpings Universitet

SE-581 83 Linköping, Sweden anbjo@mai.liu.se

Jana Björn

Department of Mathematics

Linköpings Universitet

SE-581 83 Linköping, Sweden

jabjo@mai.liu.se

Mikko Parviainen

Institute of Mathematics

Helsinki University of Technology

P.O. Box 1100, FI-02015 Helsinki University of Technology, Finland

Mikko.Parviainen@tkk.fi

The first two authors were supported by the Swedish Research Council. This research belongs to the European Science Foundation Networking Programme Harmonic and Complex Analysis and Applications. 